\title{
Application and Benefits of Web-Mediated Symptom Reporting for Patients Undergoing Immunotherapy: A Clinical Example
}

\author{
Fabrice Denis $^{a, c} \quad$ Bridget F. Koontz ${ }^{b} \quad$ Christophe Letellier $^{c}$ \\ alnstitut Inter-régional de Cancérologie Jean Bernard, 9 rue Beauverger, Le Mans, \\ France; 'bepartment of Radiation Oncology, Duke Cancer Institute, Durham, NC, USA; \\ 'Normandie Université - CORIA, Campus Universitaire du Madrillet, \\ Saint-Etienne du Rouvray, France
}

\begin{abstract}
Two randomized studies of symptom monitoring during chemotherapy or during second line treatment and follow-up via web-based patient-reported outcomes (PROs) was previously demonstrated to lengthen survival. We are presenting here a patient with advanced and recurrent lung cancer who was followed for 4 years by PROs for relapse and adverse events detection. We report how the web-mediated follow-up helped to detect dangerous pulmonary embolism, relapse and pseudo-progression to immunotherapy by self-reported symptom tracking and specific algorithms triggering notifications to medical team, allowing early management of events. We particularly describe how a discordance between objective clinical improvement under immunotherapy assessed by the application allowed to detect pseudo-progression on imaging and allowed maintenance of the treatment during more than 1-year, although imaging report could have led to stop an effective therapy. The progression observed in the routine imaging was indeed in clear contradiction with improvements in patient's global status as assessed by the reduced PRO-score computed from patient self-reported symptoms. The ability of e-health tools based on symptoms reporting for tumor response assessment should be assessed in trials to help physician in decision of stopping or continuing therapy.

(C) 2018 The Author(s)

Published by S. Karger AG, Basel
\end{abstract}




\section{Case Reports in Oncology}

\begin{tabular}{l|l} 
Case Rep Oncol 2018;11:763-768 \\
\hline DOI: 10.1159/000494829 & $\begin{array}{l}\text { ( 2 } 2018 \text { The Author(s). Published by S. Karger AG, Basel } \\
\text { www.karger.com/cro }\end{array}$
\end{tabular}

Denis et al.: Application and Benefits of Web-Mediated Symptom Reporting for Patients Undergoing Immunotherapy: A Clinical Example

\section{Case Report}

Mobile applications can be used for improving the therapy workflow in oncology [1]. Patient self-reported outcomes transmitted by connected devices are of growing interest in cancer clinical care settings since randomized trials in oncology recently reported survival benefit [2-7]. Web-mediated self-reported symptoms allow to quickly inform the physician about patient's evolution and, consequently, early detection of toxic therapies, dangerous medical outcomes, relapse, and necessity for supportive care. We have previously shown an improvement in early diagnosis of recurrence, quality of life, and overall survival in a randomized phase III trial using a web-mediated symptom tracking platform (Moovcare ${ }^{\mathrm{TM}}$ ) [2].

Self-reported symptom tracking can also accurately assess a patient's response to treatment [6]. New therapies such as immunotherapy or targeted therapies can often present with "flares" on imaging that are difficult to distinguish from disease progression. Here, we report the case of a patient using the Moovcare ${ }^{\mathrm{TM}}$ web-mediated follow-up whose changes in symptoms were tracked during immunotherapy.

The patient, a man born in 1949, had a left pneumonectomy in 2005 (month M0) for a cT3 squamous cell carcinoma (Table 1). A trifocal relapse occurred in his right lung at M72 and the patient was treated with stereotactic radiotherapy. Another relapse occurred in this right lung in M107: the patient received paclitaxel $\left(175 \mathrm{mg} / \mathrm{m}^{2}\right)$ associated with carboplatin AUC 6 for 6 successive 21 day cycles. After partial response, he was initiated on maintenance erlotinib (150 mg/day). At M116, this patient was enrolled in a phase III randomized trial of webmediated follow-up for assessing the relevance of weekly reported symptoms to the medical team [2]. The patient weekly self-scored 12 symptom changes in weight, anorexia, asthenia, cough, dyspnea, pain [2]. The follow-up intervention was designed such that a change determined by algorithm to be at high risk would initiate an alert to the research team and treating provider.

Alerted by patient's self-reported outcomes noting increasing dyspnea (M117), the patient was evaluated and diagnosed with a pulmonary embolism. The patient was treated with Tinzaparin for 6 months and clinical improvement of his dyspnea was observed using the web-mediated follow-up (Fig. 1). No tumor progression was observed by routine imaging (every 3 months) and the web-mediated follow-up was maintained.

Fatigue and anorexia were detected at M141 from the weekly self-reported outcomes. Imaging showed a tumor progression in his right lung without other metastasis (S2 in Fig. 1). Nivolumab $(3 \mathrm{mg} / \mathrm{kg} / 2$ weeks) was therefore initiated. Patient continued to use the web-mediated follow-up. An imaging was performed to assess tumor response at M142: although the tumor size increased between months M141 and M142 (S3 in Fig. 1), patient's weekly reported outcomes showed an improvement of patient's anorexia, dyspnea and fatigue, suggesting a good response to treatment. Therefore, despite the suggestion of progression on imaging, the treatment with nivolumab was maintained. Follow-up imaging performed at M144 (S4 in Fig. 1) confirmed tumor regression. Per the web-mediated tracking, symptoms and weight improved then stabilized. The treatment with Nivolumab was therefore maintained and is ongoing.

Tumor pseudo-progression is commonly associated with an increase of tumor size on imaging contradictory to clinical response to treatment. Pseudo-progression has been quite often reported and noted during immunotherapy in metastatic melanoma since affecting 10 to 


\section{Case Reports in Oncology}

Case Rep Oncol 2018;11:763-768

DOI: $10.1159 / 000494829$

C 2018 The Author(s). Published by S. Karger AG, Base www.karger.com/cro

Denis et al.: Application and Benefits of Web-Mediated Symptom Reporting for Patients Undergoing Immunotherapy: A Clinical Example

$15 \%$ of patients treated by PD-1 inhibitors for metastatic malignant melanoma [8-9]. It has also been reported in lung cancer patients receiving immunotherapy [10]. In fact, when a treatment is initiated, it may appear that immune cells are well stimulated and migrate to tumor, thus increasing its size: tumor pseudo-progression is therefore not associated with an increase of the population of tumor cells but rather with an increase of the immune cell population within the tumor boundaries. If mis-diagnosed as progression, this feature can lead to an interruption of an effective therapy.

In the present case, an indisputable pseudo-progression was observed after nearly 6 weeks of immunotherapy. The progression observed in the routine imaging was in clear contradiction with improvements in patient's global status as assessed by the reduced PRO-score computed from patient self-reported symptoms. Commonly a lack of response to treatment for a cancer relapse in lung is associated with an increase in symptoms such as dyspnea, asthenia and/or anorexia [11]. Although tumor size progression was observed in the imaging, because the symptoms necessarily associated with a bad response to treatment (dyspnea, weight loss, cough, pain, etc.) were lacking, the physician maintained the immunotherapy. This choice was supported by the very good sensitivity and specificity for detecting cancer relapse using the web-mediated follow-up Moovcare ${ }^{\mathrm{TM}}$ [2]. The imaging performed 2 months later (M144) showed partial response and maintenance of symptoms benefits, thus confirming the adopted strategy. The appreciation of this clinical situation would not have been so accurate without the well-established reliability of the web-mediated follow-up. Moreover, Moovcare ${ }^{\mathrm{TM}}$ provides in a single sight (see Fig. 1) a synthetic and accurate account for the time evolution in symptoms, remembering symptoms that occurred two months earlier.

Another advantage of the web-mediated follow-up is that routine imaging is planned once a year when there is no alarm triggered by the web-application; the total number of imaging is thus reduced compared to a standard follow-up. In the present case, during the 36 months he had routine follow-up (between M72 and M116), the patient received 8 clinically non-motivated imaging (2 CT scan and 6 PET) and after his inclusion in the phase III trial (34 months starting in M116) he only received 6 clinically non-motivated CT scans. In both cases, he received 2 imaging associated either with a relapse or with a serious pathology (pulmonary embolism). The number of non-motivated imaging was therefore reduced by $25 \%$ for nearly the same duration. Such a reduction in non-motivated imaging may even be decreased further in the future when more experience will be acquired with the use of Moovcare ${ }^{\mathrm{TM}}$.

Immunotherapy is usually a well-tolerated treatment and its low toxicity does not affect the symptoms as assessed by patients' reported outcomes. Indeed the web-mediated followup can also help to early detect the toxicity or conversely to confirm the good response to treatment such as chemotherapy (Fig. 2) or immunotherapy. It could be a useful complementary tool to common approaches such as Immune Related-RECIST criteria that were designed to help clinicians for continuing or not immunotherapy in patients for who information provided by imaging is not sufficient to have a clear diagnostic. Tolerance to treatment or benefit can be reported by patient but it has necessarily a subjective component. Monitoring KRASmutated ctDNA may also help to discriminate pseudo-progression from true progression during anti-PD-1 treatment of lung adenocarcinoma as reported recently by Guibert et al. [10], but this requires biopsy. Moovcare ${ }^{\mathrm{TM}}$ thus appears as a useful noninvasive alternative or, at least, as a powerful additional tool since in a randomized trial we already showed that this 
Denis et al.: Application and Benefits of Web-Mediated Symptom Reporting for Patients Undergoing Immunotherapy: A Clinical Example

approach helps to early detect relapse or deleterious medical complication and, consequently, leads to survival improvement in lung cancer [2].

\section{Statement of Ethics}

The authors have no ethical conflicts to disclose.

\section{Disclosure Statement}

The authors have no conflicts of interest to declare.

\section{References}

1 Kessel KA, Vogel MM, Schmidt-Graf F, Combs SE. Mobile Apps in Oncology: A Survey on health care professionals' attitude toward telemedicine, mHealth, and oncological apps. J Med Internet Res. 2016 Nov;18(11):e312.

2 Denis F, Lethrosne C, Pourel N, Molinier O, Pointreau Y, Domont J, et al. Randomized Trial Comparing a WebMediated Follow-up With Routine Surveillance in Lung Cancer Patients. J Natl Cancer Inst. 2017 Sep;109(9):djx029.

3 Denis F, Viger L, Charron A, Voog E, Letellier C. Detecting lung cancer relapse using self-evaluation forms weekly filled at home: the sentinel follow-up. Support Care Cancer. 2014 Jan;22(1):79-85.

4 Denis F, Viger L, Charron A, Voog E, Dupuis O, Pointreau Y, et al. Detection of lung cancer relapse using selfreported symptoms transmitted via an internet web-application: pilot study of the sentinel follow-up. Support Care Cancer. 2014 Jun;22(6):1467-73.

5 Denis F, Yossi S, Septans AL, Charron A, Voog E, Dupuis O, et al. Improving survival in patients treated for a lung cancer using self-evaluated symptoms reported through a web application. Am J Clin Oncol. 2017 Oct;40(5):464-9.

6 Basch E, Deal AM, Kris MG, Scher HI, Hudis CA, Sabbatini P, et al. Symptom monitoring with patient-reported outcomes during routine cancer treatment: A randomized controlled trial. J Clin Oncol. 2016 Feb;34(6):55765.

7 Oldenmenger WH, Baan MA, van der Rijt CC. Development and feasibility of a web application to monitor patients' cancer-related pain. Support Care Cancer. 2018 Feb;26(2):635-42.

8 Hodi FS, Sznol M, Kluger HM, McDermott DF, Carvajal RD, Lawrence DP, et al. Long term survival of ipilimumab-naive patients with advanced melanoma treated with nivolumab (anti-PD-1, BMS-936558, ONO4538) in a phase I trial. J Clin Oncol. 2014;15 abstr 9002.

9 Hodi SF, Ribas A, Daud A, Hamid O, Robert C, Kefford R, et al. Evaluation of immune-related response criteria (irRC) in patients (pts) with advanced melanoma (MEL) treated with the anti-PD-1 monoclonal antibody MK-3475. J Clin Oncol. 2014;15 abstr 3006.

10 Guibert N, Mazieres J, Delaunay M, Casanova A, Farella M, Keller L, et al. Monitoring of KRAS-mutated ctDNA to discriminate pseudo-progression from true progression during anti-PD-1 treatment of lung adenocarcinoma. Oncotarget. 2017 Jun;8(23):38056-60.

11 Walsh D, Rybicki L, Nelson KA, Donnelly S. Symptoms and prognosis in advanced cancer. Support Care Cancer. 2002 Jul;10(5):385-8. 


\section{Case Reports in Oncology}

\begin{tabular}{l|l} 
Case Rep Oncol 2018;11:763-768 \\
\hline DOI: 10.1159/000494829 & $\begin{array}{l}\text { (c) 2018 The Author(s). Published by S. Karger AG, Basel } \\
\text { www.karger.com/cro }\end{array}$
\end{tabular}

Denis et al:: Application and Benefits of Web-Mediated Symptom Reporting for Patients Undergoing Immunotherapy: A Clinical Example

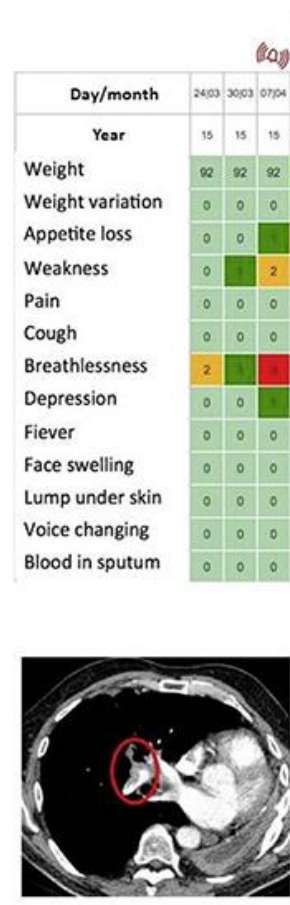

$\mathrm{S}_{1}$

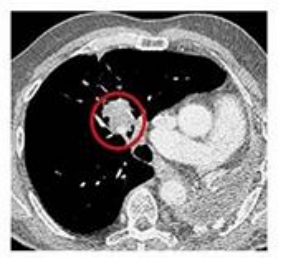

$\mathrm{S}_{2}$

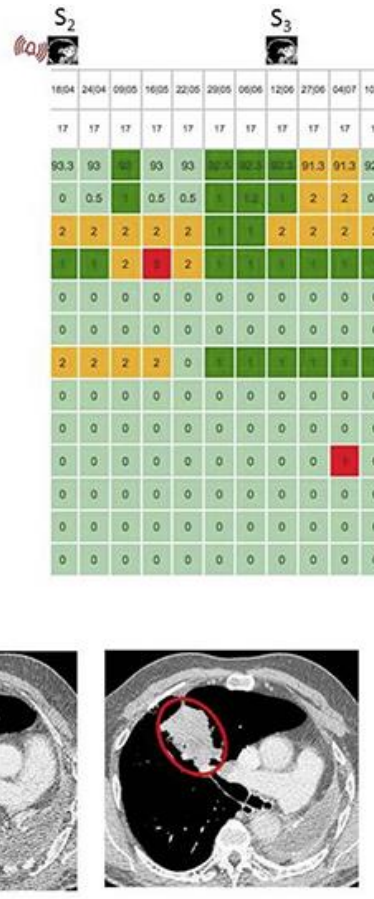

$\mathrm{S}_{3}$

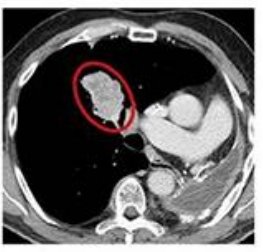

$\mathrm{S}_{4}$

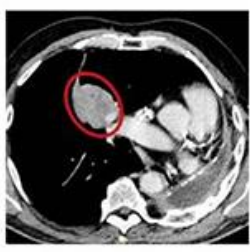

$\mathrm{S}_{5}$

Fig. 1. Screenshots of the synthetic representation (never seen by the patient) of the time evolution of patient's symptoms from his weekly completed forms. Scores are converted into a color scale as follows. $0=$ light green (no problem), 1 = dark green (mild problem), 2 = yellow (medium problem), and 3 = red (important problem). In the present case, the web-application sent an alert (M117) to the medical staff (see the red signal) subsequently to an important dyspnea. A phone call confirmed the symptoms and an anticipated CT scan (S1) was performed the same day, and revealed a massive pulmonary embolism. Treatment was quickly initiated and clinical improvement was reported (breathlessness and weakness returned to light and dark green, respectively after 2-weeks with anti-coagulation). Two years later (M141), an alarm (see the red signal) triggered an imaging (S2) which revealed a relapse; and nivolumab was initiated. An imaging (S3) showed a pseudo-progression - as confirmed by an imaging (S4), contrary to what was indicated by the color map. The next imaging (S5) showed partial response and clinical benefit was maintained (see the color map for months M145 to M147). 


\section{Case Reports in Oncology}

\section{Case Rep Oncol 2018;11:763-768}

DOI: $10.1159 / 000494829$

2018 The Author(s). Published by S. Karger AG, Basel www.karger.com/cro

Denis et al.: Application and Benefits of Web-Mediated Symptom Reporting for Patients Undergoing Immunotherapy: A Clinical Example

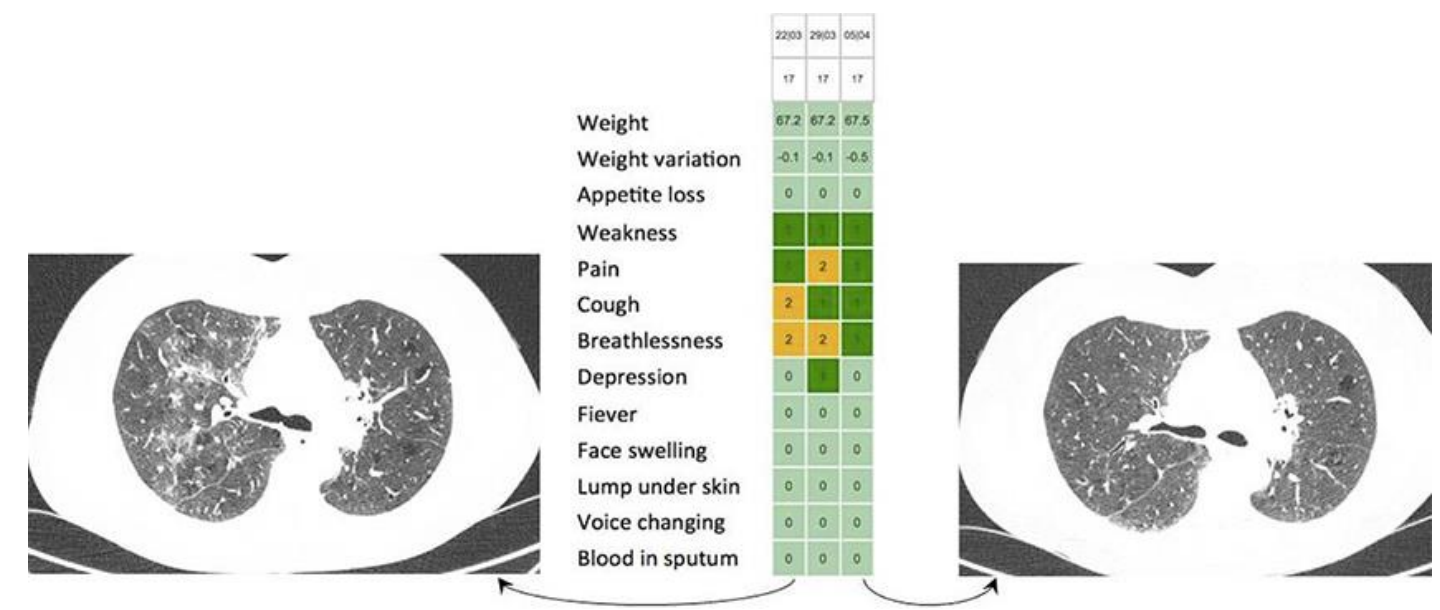

Fig. 2. Example of immunotherapy lung toxicity reported in another patient under Avelumab. Moderate cough and dyspnea without fever were reported by the patient. An unplanned CT-scan was quickly performed and suggested an auto-immune pneumopathy. Steroids were used and clinical improvement appeared in the next weeks as confirmed by a CT-scan.

Table 1. Main events and web-mediated notifications

- $\quad$ August 2005 (M0) left pneumonectomy

- $\quad$ December 2011 (M72) trifocal relapse in the right lung treated by SRS

- December 2014 (M108) 2nd relapse in the right lung treated by chemotherapy

- March 23, 2015 (M116) Inclusion in the Phase III trial

- April 13, 2015 (M117) web-alert due to a pulmonary embolism

- April 18, 2017 (M141) web alert due to a tumor progression in the right lung treated by immunotherapy

- $\quad$ May 30, 2017 (M142) tumor apparent progression (routine imaging)

- July 26, 2017 (M144) partial response to treatment

- January 2018 (M150) follow-up ongoing 